\title{
Depicting developing trend and core knowledge of hip fracture research using bibliometric and visualized analysis
}

\section{Guanrong Peng}

Yudu People's Hospital

\section{Zhenhua Guan}

Yudu People's Hospital

\section{Yunfei Hou}

Peking University People's Hospital

Jiaxiang Gao

Peking University People's Hospital

\section{Wenqun Rao}

Yudu People's Hospital

\section{Xianyun Yuan}

Yudu People's Hospital

Jiusheng Guo

Yudu People's Hospital

\section{Xiaohua Huang}

Nantong Tumor Hospital

Zhangrong Zhong ( $\sim 15901186908 @ 139 . c o m$ )

Yudu People's Hospital

Jianhao Lin

Peking University People's Hospital

\section{Research article}

Keywords: hip fracture, bibliometrics, visualized analysis, developing trend

Posted Date: August 13th, 2020

DOI: https://doi.org/10.21203/rs.3.rs-56742/v1

License: (c) (1) This work is licensed under a Creative Commons Attribution 4.0 International License.

Read Full License 


\section{Abstract}

Background Among all osteoporotic fractures, hip fracture is common and carries high morbidity and mortality. We aim to analyze the present status and worldwide trends of hip fracture research in various clinical aspects.

Methods Publications from 2010 to 2019 were retrieved from Web of Science database and analyzed through bibliometric methodology. The software VOS viewer was utilized for visualized analysis.

Results Totally, 6139 publications were included, and publications increased annually, from 152 (2000) to 592 (2019). The USA has made huge contributions with the most publications, the highest H-index, and citations. Osteoporosis Internationa/ has published the most papers. Leading researchers, contributing institutions, their cooperation relationships and scientific masterpieces have been identified. Research could be divided into five clusters: "mortality", "surgical management", "rehabilitation", "osteoporosis" and "epidemiology". A clear developing trend was described, which initiated from fracture epidemiology and prevention, transitioned to perioperative management, orthogeriatric care and patient safety, and then to functional recovery, disease burden along with national audit and initiatives in recent time.

Conclusions Hip fractures resulted in conditions that extend far beyond orthopaedics, concerning epidemiology \& preventive medicine, internal medicine \& endocrinology, as well as critical care and gerontology. And a growing volume of publications in the future could be expected.

\section{Background}

With ageing process develops around the world, hip fracture has been becoming a vital concern. The global amount of hip fractures is anticipated to boost from 1.26 million in 1990 to 4.5 million by the year 2050. Even though the age-standardised incidence is slowly decreasing in a lot of nations, this is much outweighed by the process of population ageing ${ }^{1}$.

Among all osteoporotic fragility fractures, hip fracture carries the highest morbidity and mortality ${ }^{2}$. Allcause and excess mortality after hip fracture was greater than age-matched controls even over two decades of follow-up ${ }^{3}$. Fracture survivors encountered substantially worse mobility, independence in function, overall health, and quality of life. To our best knowledge, the worldwide investigation trends in hip fractures has not been well analyzed yet which is crucial to study indeed.

Bibliometric analysis is a feasible strategy to summarize and anticipate the research trends qualitatively and quantitatively, by evaluating the studies of major authors, journals, institutes, and nations ${ }^{4}$. Additionally, it makes contributions to clinical policy-making and guidelines development ${ }^{5}$. The objective of this study was to adequately analyze the present status and worldwide trends of hip fracture research in various clinical aspects.

\section{Methods}




\section{Data source}

While many databases could satisfy the need for analysis in a worldwide level ${ }^{6}$, we selected the Web of Science (WoS) and Science Citation Index-Expanded for this evaluation, and they cover over 12,000 scientific international journals of greatest impact and quality, offering detailed information of publications ${ }^{7}$.

\section{Search strategy}

The search strategy was as follows: (title $=$ hip And title $=$ fracture* $)$. We excluded pathological fractures caused by bone tumors and fractures following any types of hip arthroplasty including, total hip arthroplasty, hemiarthroplasty, and resurfacing arthroplasty. The time period of publications was centered on the current 20 years from 2000 to 2019 , and the document types exclusively contained reviews(355) and articles(5784). Retrieval work was carried out in the same day (on June 21, 2020) to avoid the variations because of daily updates.

\section{Data extraction}

The information of all identified publications, including title, author, publication year, contributing nations, affiliations, journal, keywords and abstract, was downloaded from the WoS database. Two authors independently browsed and then extracted data from the eligible publications.

\section{Bibliometric analysis}

The basic characteristics of publications were retrieved by the intrinsic function of WoS. The H-index is described as the value according to a scholar or scientist who has published $\mathrm{H}$ papers, each of which has been cited by other studies for no less than $\mathrm{H}_{\text {times }}{ }^{8}$. Therefore, the $\mathrm{H}$-index could reflect the number of both publications and their relevant citations, to evaluate the productivity of the author and the impact of the published research ${ }^{9}$.

\section{Visualized analysis}

VOS viewer (Leiden University, Leiden, The Netherlands) is a program for creating and visualizing bibliometric networks ${ }^{10}$. In this particular study, VOS viewer was used for coauthorship, cocitaion and cooccurrence analysis. In the network map developed by VOS viewer, various nodes represented different elements, for example, authors, countries, institution, and keywords. The size of the nodes reflected the amount of the publications or frequency ${ }^{11}$. The links between nodes represented the associations including coauthorship or co-occurrence, while the color of the node/lines reflected diverse clusters or years $^{12}$. The strength of the link was presented as total link strength (TLS).

Coauthorship analysis illustrates the connection among items in line with the number of coauthored papers, which is an effective tool to evaluate collaboration trends and to identify leading researchers, nations, and organizations ${ }^{13}$. Co-occurrence analysis illustrates the connection of key words according to 
the quantity of publications where they were found together ${ }^{14}$. The analysis could explore the popular aspects and research directions, and also it happens to be a crucial indicator to observe developments in one specific area. A repeated co-occurrence analysis was conducted using a second data set whose time period focused on 2018-2020 while other conditions (selected database, search strategy, exclusion criteria and document types) remained the same in order to capture the hot topics of hip fracture research in the latest several years. The keywords with high occurrences were compared with those generated from the analysis using the primary data set whose time period focused on 2000-2019.

\section{Results}

\section{Quantity of global publications}

A total of 6139 publications (reviews(355) and articles(5784)) were ultimately included after using the search criteria. Of the past 2 decades, the quantity of publications has increased yearly, from 152 (2000) to 592 (2019), shown in Table 1. Most of the investigations were published in 2019 (592, 9.6\%). A total of 86 nations and regions have published relevant articles/review, and the countries that had made the greatest contributions were presented in Table 1. Among them, the USA contributed with the most publications $(1766,28.8 \%)$, followed by UK $(1269,20.7 \%)$, China $(615,10.0 \%)$, and Canada $(487,7.9 \%)$. A distribution world map of hip fracture research was shown in Fig. 1.

Table 1

The quantity of hip fracture research in terms of year and country

\begin{tabular}{|llll|}
\hline \multicolumn{2}{|l}{ Year (Publications) } & \multicolumn{2}{l|}{ Country (Publications) } \\
\hline $2019(592)$ & $2009(272)$ & USA (1766) & Netherlands (263) \\
\hline $2018(496)$ & $2008(228)$ & UK (1269) & Denmark (230) \\
\hline $2017(506)$ & $2007(214)$ & China (615) & Norway (221) \\
\hline $2016(466)$ & $2006(217)$ & Canada (487) & France (182) \\
\hline $2015(407)$ & $2005(215)$ & Sweden (379) & Israel (174) \\
\hline $2014(393)$ & $2004(170)$ & Australia (365) & Finland (167) \\
\hline $2013(378)$ & $2003(163)$ & Spain (311) & South Korea (166) \\
\hline $2012(331)$ & $2002(162)$ & Italy (282) & Switzerland (149) \\
\hline $2011(349)$ & $2001(113)$ & Japan (271) & Turkey (124) \\
\hline $2010(315)$ & $2000(152)$ & Germany (267) & India (101) \\
\hline
\end{tabular}

\section{Quality of the publications from each country/region}


The total number of citations and the H-index reflect the quality of the publications and academic impact of one country ${ }^{15}$. The USA had the highest number of total citations $(64,086)$, while the UK ranked the second (28505), then followed by Canada (17001), Sweden (13387), and Australia(11386). An exactly same trend was present for the H index: USA(118), UK (76), the Canada (61), Sweden (57), and Australia (53). Publications from Switzerland had the most average citation frequency (45.46), then followed by France(41.33), Netherlands (40.63), USA (38.68), Canada (36.64), and Denmark (36.58).

\section{Analysis of global publications}

\section{Journals}

The Osteoporosis International published 459 articles/reviews, outranking other journals with the most publications. Injury-International Journal Of the Care Of the Injured positioned the second, with 347 publications. Furthermore, there were 165 papers published in Journal of Bone and Mineral Research, 157 in Journal of Orthopaedic Trauma and 134 in Journal of the American Geriatrics Society on the hip fracture field. The top 10 journals with the most publications were listed in Table 2.

Table 2

Leading journals, authors, and institutions of publications related to hip fracture research

\begin{tabular}{|lll|}
\hline Journal (Publications) & Author (Publications) & Institution (Publications) \\
\hline OSTEOPOROSIS INT (459) & Magaziner J (93) & Univ Maryland (145) \\
\hline Injury (347) & Parker MJ (79) & Univ Pittsburgh (112) \\
\hline J BONE MINER RES (165) & Cauley JA (58) & Univ California San Francisco (109) \\
\hline J ORTHOP TRAUMA (157) & Di Monaco M (48) & Karolinska Inst (101) \\
\hline J AM GERIATR SOC (134) & Cooper C (44) & Univ Oxford (99) \\
\hline J ARTHROPLASTY (128) & Kanis JA (42) & Harvard Univ(98) \\
\hline BONE (116) & Cummings SR (41) & Univ Toronto (97) \\
\hline J BONE JOINT SURG AM (113) & Bhandari M (40) & Tel Aviv Univ (91) \\
\hline INT ORTHOP (109) & Ha YC (40) & Univ Oslo (88) \\
\hline ARCH ORTHOP TRAUM SU (93) & Ensrud KE (39) & McMaster Univ (86) \\
\hline
\end{tabular}

\section{Research orientation}

The top 10 research orientations related to hip fracture were in Fig. 2. By far the most predominant areas of research were orthopedics (2108 papers), surgery (1184 papers), sport science (1586 papers), engineering (432 papers), and general internal medicine (589 papers), respectively.

\section{Authors}

The top 10 authors with the greatest number of publications were listed in Table 2, totally these authors have published 1151 articles/reviews throughout the last 20 years. Magaziner $\mathrm{J}$ from the USA outranked 
other researchers with 93 publications, followed by Parker MJ from UK with 79 papers, and Cauley JA from USA with 58 papers. It is noteworthy that we included all of the authors within the analysis, regardless of the authors' relative contribution (1st author, correspondence author, or co-author) towards one single research.

\section{Institution output}

University of Maryland had the greatest number of publications with 145 papers, followed by the University of Pittsburgh (112 papers) and then the University of California, San Francisco (109 papers). Table 2 presented the top 10 institutions with the most publications.

\section{Top 10 most-cited articles \& Top 10 articles with largest annual citations}

The mean number of citations per publication was 25.9 times. Table 3 demonstrated the top 10 mostcited articles regarding hip fracture. The most highly cited article was "Effect of risedronate on the risk of hip fracture in elderly women" published in the New England Journal of Medicine (NEJM) by McClung et al $^{16}$, with 1321 citations on WOS. Table 4 demonstrated the top 10 articles regarding hip fracture with the largest annual citations, among which the article "Zoledronic acid and clinical fractures and mortality after hip fracture" published in the NEJM by Lyles et al ${ }^{17}$ with 75.5 annual citations, ranked the 1 st . 
Table 3

Top 10 most-cited publications in hip fracture research

\section{Rank Title of the publication/ First author/ Publishing year/ Publishing journal}

Citation

rate

1 Effect of risedronate on the risk of hip fracture in elderly women/ McClung/ 2001/

The New England Journal of Medicine

2 Zoledronic acid and clinical fractures and mortality after hip fracture/ Lyles/ 2007/ The New England Journal of Medicine

3 Predictive value of BMD for hip and other fractures/ Olof/ 2005/ Journal of Bone and Mineral Research

$4 \quad$ Long-term proton pump inhibitor therapy and risk of hip fracture/ Yang/ 2006/ The Journal of the American Medical Association

5 Incidence and Mortality of Hip fractures in the United States/ Braur/ 2009/ The Journal of the American Medical Association

6 The use of clinical risk factors enhances the performance of BMD in the prediction of hip and osteoporotic fractures in men and women/ Kanis/2007/ Osteoporosis International

7 Reducing delirium after hip fracture: A randomized trial/ Marcantonio/ 2001/ Journal of American Geriatric Society

8 Effect of comorbidities and postoperative complications on mortality after hip fracture in elderly people: prospective observational cohort study/ Roche/ 2005/ British Medical Journal

9 Meta-analysis: Excess Mortality After Hip fracture Among Older Women and Men/ Patrick/ 2010/ Annals of Internal Medicine worldwide/ Kanis/ 2012/ Osteoporosis International
1321

1057

866

745

695

690

689

658

600

530 
Table 4

Top 10 publications with the largest annual citations

\section{Rank Title of the publication/ First author/ Publishing year/ Publishing journal}

Annual citations

$1 \quad$ Zoledronic acid and clinical fractures and mortality after hip fracture/ Lyles/ 2007/ The New England Journal of Medicine

2 Effect of risedronate on the risk of hip fracture in elderly women/ McClung/ 2001/ The New England Journal of Medicine

3 A systematic review of hip fracture incidence and probability of fracture worldwide/ Kanis/ 2012/ Osteoporosis International

4 Incidence and Mortality of Hip fractures in the United States/Braur/ 2009/ The Journal of the American Medical Association

$5 \quad$ Meta-analysis: Excess Mortality After Hip fracture Among Older Women and Men/ Patrick/ 2010/ Annals of Internal Medicine

6 Predictive value of BMD for hip and other fractures/ Olof/ 2005/ Journal of Bone and Mineral Research

7 Long-term proton pump inhibitor therapy and risk of hip fracture/ Yang/ 2006/ The Journal of the American Medical Association prediction of hip and osteoporotic fractures in men and women/ Kanis/2007/ Osteoporosis International

9 Effect of comorbidities and postoperative complications on mortality after hip 41.13 fracture in elderly people: prospective observational cohort study/ Roche/ 2005/ British Medical Journal 2011/ Osteoporosis International

\section{Visualized analysis}

\section{Coauthorship analysis}

\section{Authors}

As presented in Fig. 3a, a total of 480 authors with a minimum limitation of more than 5 publications were identified and analyzed via VOS viewer. The top five authors with greatest TLS were shown as follows: Magaziner J (TLS = 372 times), Cauley JA (TLS = 189 times), Ensrud K (TLS = 127 times), Cumming $S(T L S=124$ times), and Orwig D (TLS = 118 times).

\section{Countries and regions}

A total of 54 countries and regions with a minimum limitation of more than 5 publications were identified and analyzed by using VOS viewer. The top five country and regions with largest TLS were presented as 
follows: the USA (TLS = 683 times), the UK (TLS = 595 times), Canada (TLS = 341 times), Sweden (TLS = 287 times), and Netherlands (TLS = 280 times) as shown in Fig. 3b.

\section{Institutions}

Presented in Fig. 3c, 622 institutions were included with a minimum limitation of more than 5 publications. Moreover, the University of Pittsburgh (TLS = 395 times), University of Maryland (TLS = 332 times), University of California San Francisco (TLS $=318$ times), Harvard University (TLS $=260$ times), and McMaster University (TLS = 249 times) were the top five institutions with greatest TLS.

\section{Co-occurrence analysis}

The keywords which had been utilized over five times among included publications, were recognized and analyzed via VOS viewer. As presented in Fig. 4a by different colors, the 1458 included keywords could be divided into approximately 5 clusters: "mortality study", "surgical management study", "rehabilitation study", "osteoporosis study", and "epidemiology study". In detail, in the cluster of "mortality study", the main and frequent keywords were morbidity, survival, operative delay, complications and blood loss. As for the "surgical management study" cluster, the frequent keywords were arthroplasty, fixation, follow-up, outcomes, and failure. In the "rehabilitation study" cluster, keywords include nursing home, geriatric rehabilitation, cognitive impairment and delirium. As for the "osteoporosis study" cluster, the prominent keywords were bone mineral density (BMD), aging, risk prediction, and trabecular bone. In the cluster of "epidemiology study", the frequent keywords were incidence, rates, population, and risk factors. The overlay visualization map of the co-occurrence analysis, with items noted with different colors in accordance with the average time period when the keywords occurred ${ }^{18}$ was illustrated in Fig. $4 \mathrm{~b}$. The color blue indicated the keywords appeared earlier, while the color red stood for the later occurrence. Before 2010, keywords included "rehabilitation", "surgery", "morbidity", "mortality", "complications", coded as blue color, occurred earlier, as they are key and elemental aspects in hip fracture management and research; while after 2010, as the research conducted in a more detailed manner, keywords including "timing/delay of surgery", "transfusion", "registry", "mobile" and etc. started to occur.

A repeated co-occurrence analysis using a second data set whose time period focused on 2018-2020 demonstrated keywords with high occurrence in the latest few years, which were compared with those generated using the primary data set. The comparisons of the key words were illustrated in Table 5. From 2018 to present, the keyword "surgery", "outcomes", "elderly-patients", "management", "complications" occurred more often, as the rankings of which has been improved. 
Table 5

Top 10 key words with the highest occurrences in different time period:

$2018-2020$ vs $2000-2019$

\begin{tabular}{|lll|}
\hline Rank & $\mathbf{2 0 1 8 - 2 0 2 0}$ & $\mathbf{2 0 0 0 - 2 0 1 9}$ \\
\hline 1 & mortality & mortality \\
\hline 2 & surgery* & osteoporosis \\
\hline 4 & risk & risk \\
\hline 5 & outcomes* & women \\
\hline 6 & elderly-patients* & surgery \\
\hline 8 & management* & Bone-mineral density \\
\hline 9 & women & Elderly-patients \\
\hline 10 & risk-factors & epidemiology \\
\hline$*:$ Higher rankings compared to which at the time period 2000-2019 & Risk-factors \\
\hline
\end{tabular}

\section{Discussion}

Bibliometric and visualized analysis are regarded as appropriate tools for describing the present status and predicting the future trends concerning the research of interest. In this study, the current status and global trends of hip fracture research were delineated. The quantity of publications has gradually increased yearly. Leading researchers, contributing institutions, countries and their cooperation relationship have been identified, and important publications with high-citations highlighted.

Utilizing co-occurrence cluster analysis, we presented a network map of co-occurrence relationship by analyzing the keywords of all the included studies. Totally, five potential research orientations were identified and keywords as mortality, osteoporosis, risk, elderly-patients and etc. were highlighted with bigger icons. These results suggested that hip fractures, especially in older individuals, lead to conditions that extend far beyond the orthopaedic injury, with consequences in the aspects of epidemiology \& preventive medicine, internal medicine \& endocrinology, as well as critical care and gerontology. This is also confirmed in Fig. 2. Using the overlay visualization map of the co-occurrence analysis, different colors represented the relevant year of publication. Nodes of various colors (from blue to red) could all be found with substantial densities in the five clusters, which suggested a pattern of balanced development existed in these five investigation directions respectively during the past decade. Specifically, a pattern of balanced development may exist within the field of hip fracture, accompanied with inherent alterations of hotspots in each sub-orientation. Additionally, each direction itself was also experiencing the changes of research hotspot, suggesting a diversified developing trend. Also by using this network map together with 
other bibliometric information, we had chances to further shed light on the growing trends in the past, present and the future.

The number of citations amassed by a publication could be employed as a surrogate marker of the impact made within its field ${ }^{19}$. Interestingly, $90 \%$ of the leading 10 hip fracture studies with the largest overall citation count feature in the top 10 for annual citations, implying both historical significance as well as significance to current medical practice. Most of these highly-cited studies were published before 2010 , as prior studies have longer time period of citation accumulation inherently. They mainly focused on osteoporosis management, fracture epidemiology and prevention, as well as perioperative management and patient safety.

\section{Fracture prevention and medication therapy}

The most-cited report was published by Dr. McClung in 2001 describing the protective effects of risedronate which substantially minimized hip fracture risk among aged females with established osteoporosis but not among those with risk factors apart from lower BMD, i.e. poor gait or fall propensity ${ }^{16}$. From this large trial (9331 female patients), the results demonstrated the value of BMD measurements in identifying women for whom medication therapy to prevent hip fracture is adequate. Similarly, a double-blinded randomized controlled trial (RCT) discovered that an annual infusion of zoledronic acid within 90 days following surgical fixation of a fragility hip fracture was associated with a reduction of a new clinical fracture rate together with improved survival ${ }^{17}$. While conversely, a nested case-control study carried out utilizing the General Practice Research Database (1987-2003) in UK discovered that long-term proton pump inhibitors therapy, especially at high doses, was associated with an elevated hip fracture risk ${ }^{20}$.

\section{Epidemiology}

On fracture risk: Other research centered on hip fracture epidemiology. In the United States, hip fracture rates and consequent mortality among individuals 65 years and older were decreasing along with the usage of bisphosphonates and comorbidities among patients with hip fractures had increased ${ }^{21}$. Hip fracture risk could be predicted by BMD and clinical risk factors(CRFs). The prediction model, with the combined use of CRFs and BMD than BMD alone, could be improved with a greater gradient of risk (risk ratio/standard deviation change in risk score) from $3.7 / S D$ to $4.2 / S^{22}$. An additional systematic review(SR) reported that age-standardised rates of hip fracture were accessible for 63 countries $^{23}$. Additionally, there was a greater than 10 -fold variation in hip fracture risks between nations. High risk regions for men were Taiwan, Austria, USA (Caucasian), Switzerland, Norway, Sweden and Denmark. Those at low risk included Tunisia, Oceania, the Latin American countries of Ecuador and Colombia and several European countries (Spain, Poland, Romania, France and Turkey). Other countries at low risk were China, Lebanon, Philippines and the US Black population. The basic pattern of fracture likelihood in women was comparable to that in men. And discordances within classification were fairly few. Five countries coded as low risk in men were at intermediate risk for women (Poland, New Zealand, Romania, 
France and Turkey). Seven countries coded as moderate risk in men were coded at high risk in women (Japan, Belgium, Singapore, Canada, Malta, UK and Slovakia) ${ }^{23}$.

On mortality: a meta-analysis found that older adults have a 5- to 8-fold higher risk for all-cause mortality throughout the first 3 months after hip fracture. Excessive annual mortality persisted for both women and men even after 10 years of follow up (FU), but at any given age, excessive annual mortality after hip fracture was higher in males than in females ${ }^{24}$.

\section{Perioperative management and orthogeriatric collaborated care}

A prospective observational cohort study reported that in elderly patients, the existence of three or more comorbidities would be the strongest preoperative risk factor for mortality within the 1st month after surgical procedures. Chest infection and heart failure were the most common early postoperative complications and resulted in increased mortality. These groups offer an apparent target for specialized medical evaluation ${ }^{25}$. Another RCT noted that proactive geriatrics consultation was effectively applied with good adherence after surgical procedures. It diminished delirium by over one-third, and reduced severe ones by over one-half. The trial provided strong preliminary evidence that proactive geriatrics consultation played a crucial role within the acute hospital management for hip fracture patients ${ }^{26}$.

8 of these 10 highly-cited articles were published from 2000-2009. While studies published from 2010 to 2014 , continued to put attention on patient safety and mortality, but in an extended time point of view and FU. A SR reported that the overall inpatient or 1 month mortality was $13.3 \%$, 3-6 months mortality was $15.8 \%, 1$ year $24.5 \%$ and 2 years $34.5 \%{ }^{27}$. There were strong evidence for 12 predictors, including advanced age, male gender, nursing home or facility residence, poor preoperative walking capacity, poor activities of daily living, greater American Society of Anaesthesiologists grading, multiple comorbidities, diabetes, cancer, cardiac diseases, poor psychological status, dementia and cognitive impairment ${ }^{27}$. A population-based study discovered that in 4 years of $\mathrm{FU}$, the most common causes of death were circulatory diseases, followed by dementia and Alzheimer's disease ${ }^{28}$. Besides, in this 5 years (20102014), researcher started put focus on surgery timing as well as orthogeriatric care and their impact on patient outcomes. Earlier surgery (less than $48^{29}$ or 72 hours $^{30}$ ) was associated with a reduced risk of death and lower rates of postoperative pneumonia and pressure sores among aged patients suggesting minimizing delay to improve outcomes ${ }^{30}$. A meta-analysis supported that ortho-geriatric cooperation to improve mortality after hip fracture fixation ${ }^{31}$. Immediate admission of patients aged 70 years or more to comprehensive geriatric care in a dedicated ward improved mobility at 4 months, compared with the usual orthopaedic care alone ${ }^{32}$.

\section{Patient rehabilitation, medical and economic burden}

Since 2015, research began to concentrate on patient rehabilitation, economic burden of the injury as well as national audit and registry studies. The fracture has a considerable impact on older peoples' mediumto longer-term capabilities, physical functions, quality of life and accommodation. Only $40 \%$ to $60 \%$ of 
study participants recovered their pre-fracture level of mobility and ability, while 40\%-70 \% regained their level of independence for fundamental activities of daily living. For individuals independent in self-care prior to fracture, $20 \%-60 \%$ needed support for various tasks at 1 and 2 years $\mathrm{FU}^{32}$. These results suggested that great medical and potential economic burden existed for hip fracture survivors. Medical expenses following hip fracture were high and mostly occurred in the first year after the index fracture, while suffering from a second hip fracture accounted for a great deal of the expense increase. There is a solid economic incentive to prioritise research funds towards figuring out the best strategies to prevent both index and subsequent hip fractures ${ }^{33}$.

\section{National audit and initiative}

The great burden caused by hip fracture warranted actions or projects in a greater scale, i.e. in country level. The UK National Hip fracture Database was launched in 2007 being a national collaborative, clinician-led audit initiative to enhance the hip fracture care quality, which was associated with significant improvements in care and survival of aged individuals with hip fracture in England. From 2007 to 2011, early surgery rate increased from $54.5 \%$ to $71.3 \%$, which had remained stable from 2003-2007. Thirtyday mortality fell from $10.9 \%$ to $8.5 \%$, in comparison with a smaller reduction from $11.5 \%$ to $10.9 \%$ previously. The yearly relative decrease in adjusted 30 -day mortality was $1.8 \%$ per year in the time period 2003-2007, compared with $7.6 \%$ per year over $2007-2011^{34}$.

A clear developing trend in hip fracture research in the past 2 decades could be described, which initiated from fracture epidemiology and prevention, transitioned to perioperative management, orthogeriatric care and patient safety, and then to patient rehabilitation, disease burden as well as national audit and initiatives in recent time period.

As the number of publications continuously increases and significant burden of hip fracture prevails, an underlying vigorous development of hip fracture study could be expected with a growing quantity of papers published in the next couple of years. In addition, the co-occurrence analysis could depict the developing trends and research hotspots, which might further provide the investigators with inspirations of topic selection and assist the funding agencies make profit investment plans. Through the results of the overlay visualization map in co-occurrences analysis, "timing of surgery, registry and patient mobility" accounted for larger ratios for color yellow, indicating more studies dedicated to patient safety, functional recovery and big data research after 2010. Additionally we used the second data set from 2018 to 2020 in co-occurrence analysis and identified several key words, i.e. "surgery", "outcomes", "elderly-patients", "management", "complications" with increasing occurrences in recent years shown in table 5. Given the above two similar and interrelated results, we anticipated several hotspots within hip fracture research. 1. Optimization of peri-operative management and complications prevention; 2 . Post-injury rehabilitation and care; 3 . Meta-analysis, registry and big data research.

This study inevitably has some limitations. Firstly, there are intrinsic differences between the results of bibliometric analysis and real-world study. For instance, some comparatively new publications with high 
quality may not attach sufficient attention due to lower citation frequency, while older articles have a tendency to accumulate more citations. A second limitation is the 'obliteration by incorporation' effect describing the bias created with citation analysis which occurs when particular ideas become so accepted that the most original work is no longer cited ${ }^{35}$. Also self-citing (or neglecting to cite a rival 's work) might bring in the inherent bias of' incomplete citing' and' omission bias.' Third, bias may still exist when considering the same short name or various expressions of certain authors and keywords. However in this study, with the usage of bibliometric and visualized analysis, hotspots and collaborative relationship among countries, authors, and institutions were identified, meanwhile scientific masterpieces highlighted and reviewed. These information could provide investigators a vivid general view within the academic field of hip fracture research. A time trend was depicted from its epidemiology, osteoporosis management and fracture prevention in the 1 st decade of 21 th century, to patient mortality and surgery timing in the later time period, to rehabilitation as well as national registry and audit research in the last period. These information could also guide stakeholders in priortising funding and optimizing care of hip fracture.

\section{List Of Abbreviations}

WoS: Web of Science; TLS: total link strength; NEJM: New England Journal of Medicine; BMD: bone mineral density; FU: follow up; RCT: randomized controlled trial; CRFs: clinical risk factors; SR: systematic review.

\section{Declarations}

\section{Availability of data and materials:}

All data generated or analyzed during this study are included in this

Ethics approval and consent to participate:

Not applicable.

\section{Consent for publication:}

Not applicable.

\section{Competing interests:}

The authors declare that they have no conflict of interests.

\section{Funding:}

None 
Study design: Z.Z. and J.L.

\section{References}

1 Veronese, N. \& Maggi, S. Epidemiology and social costs of hip fracture. Injury 49, 1458-1460, doi: 10.1016/j.injury.2018.04.015 (2018).

2 Kanis, J. A. Assessment of fracture risk and its application to screening for postmenopausal osteoporosis: Synopsis of a WHO report. Osteoporosis International 4, 368-381, doi:10.1007/BF01622200 (1994).

3 Von Friesendorff, M. et al. Hip fracture, mortality risk, and cause of death over two decades. 27, 2945-2953, doi: 10.1007/s00198-016-3616-5 (2016).

$4 \quad \mathrm{Pu}, \mathrm{Q}$. H., Lyu, Q. J. \& Su, H. Y. Bibliometric analysis of scientific publications in transplantation journals from Mainland China, Japan, South Korea and Taiwan between 2006 and 2015. BMJ open 6, e011623, doi:10.1136/bmjopen-2016-011623 (2016).

5 Wang B, Xing D, Zhu Y, Dong S, \& Zhao B. The State of Exosomes Research: A Global Visualized Analysis. BioMed Research International 2019, 1-10, doi: 10.1155/2019/1495130 (2019).

6 Abhaya V Kulkarni, Brittany Aziz, Iffat Shams, \& Jason W Busse. Comparisons of citations in Web of Science, Scopus, and Google Scholar for articles published in general medical journals. JAMA 302, 1092-1096, doi: 10.1001/jama.2009.1307 (2009).

7 Aggarwal, A. et al. The State of Lung Cancer Research: A Global Analysis. Journal of thoracic oncology : official publication of the International Association for the Study of Lung Cancer 11, 10401050, doi:10.1016/j.jtho.2016.03.010 (2016).

8 Bertoli-Barsotti, L. \& Lando, T. A theoretical model of the relationship between the h-index and other simple citation indicators. Scientometrics 111, 1415-1448, doi:10.1007/s11192-017-2351-9 (2017).

9 Izet Masic \& Edin Begic. Scientometric Dilemma: Is H-index Adequate for Scientific Validity of Academic's Work? Acta Inform Med 24, 228-232, doi: 10.5455/aim.2016.24.228-232 (2016).

10 van Eck, N. J. \& Waltman, L. Software survey: VOSviewer, a computer program for bibliometric mapping. Scientometrics 84, 523-538, doi:10.1007/s11192-009-0146-3 (2010).

11 Liang, C. , Luo, A. , \& Zhong, Z. Knowledge mapping of medication literacy study: A visualized analysis using CiteSpace. SAGE Open Med 6, 2050312118800199, doi: 10.1177/2050312118800199 (2018).

12 Liang, Y. D. et al. Study of acupuncture for low back pain in recent 20 years: a bibliometric analysis via CiteSpace. Journal of pain research 10, 951-964, doi:10.2147/.jpr.S132808 (2017). 
13 Fonseca, B. D. P. F. E., Sampaio, R. B., Fonseca, M. V. D. A., \& Zicker, F. Co-authorship network analysis in health research: method and potential use. Health Res Policy Syst 14, 34-43, doi: 10.1186/s12961-016-0104-5 (2016).

14 Pavlopoulos, G. A., Promponas, V. J., Ouzounis, C. A. \& Iliopoulos, I. Biological information extraction and co-occurrence analysis. Methods Mol Biol 1159, 77-92, doi: 10.1007/978-1-4939-0709-0_5 (2014).

15 Bastian, S., Ippolito, J. A. , Lopez, S. A. , Eloy, J. A. , \& Beebe, K. S. The Use of the h-Index in Academic Orthopaedic Surgery. J Bone Joint Surg Am 99, e14, doi: 10.2106/JBJS.15.01354 (2017).

16 McClung, M. R. et al. Effect of risedronate on the risk of hip fracture in elderly women. Hip Intervention Program Study Group. The New England journal of medicine 344, 333-340, doi:10.1056/nejm200102013440503 (2001).

17 Lyles, K. W. et al. Zoledronic acid and clinical fractures and mortality after hip fracture. The New England journal of medicine 357, 1799-1809, doi:10.1056/NEJMoa074941 (2007).

18 Hesketh, K. R., Catherine, L., Helen, B. \& Steven, H. Co-Occurrence of Health Conditions during Childhood: Longitudinal Findings from the UK Millennium Cohort Study (MCS). PLoS One 11, e0156868, doi: 10.1371/journal.pone.0156868 (2016).

19 Ellegaard, O. \& Wallin, J. A. The bibliometric analysis of scholarly production: How great is the impact? Scientometrics 105, 1809-1831, doi:10.1007/s11192-015-1645-z (2015).

20 Yang Y.X. , Lewis J.D., Epstein S \& Metz D.C. Long-term Proton Pump Inhibitor Therapy and Risk of Hip Fracture. JAMA 296, 2947-53, doi: 10.1001/jama.296.24.2947 (2006).

21 Brauer, C.A., Coca-Perraillon, M, Cutler. D.M. \& Rosen A.B. Incidence and Mortality of Hip Fractures in the United States. JAMA 302:1573-1579. doi: 10.1001/jama.2009.1462 (2009).

22 Kanis, J. A. et al. The use of clinical risk factors enhances the performance of BMD in the prediction of hip and osteoporotic fractures in men and women. Osteoporos Int. 18, 1033-1046. doi: 10.1007/s00198-007-0343-y (2007).

23 Kanis, J. A. et al, A systematic review of hip fracture incidence and probability of fracture worldwide. Osteoporos Int. 23, 2239-2256, doi: 10.1007/s00198-012-1964-3. (2012).

24 Haentjens, P. et al. Meta-analysis: Excess Mortality After Hip Fracture Among Older Women and Men. Ann Intern Med. 152, 380-390. doi: 10.7326/0003-4819-152-6-201003160-00008 (2010)

25 Roche, J. J., Wenn, R. T., Sahota, O. \& Moran, C. G. Effect of comorbidities and postoperative complications on mortality after hip fracture in elderly people: prospective observational cohort study. BMJ 331, 1374, doi:10.1136/bmj.38643.663843.55 (2005). 
26 Marcantonio, E. R., Flacker, J. M., Wright, R. J. \& Resnick, N. M. Reducing delirium after hip fracture: a randomized trial. Journal of the American Geriatrics Society 49, 516-522, doi:10.1046/j.15325415.2001.49108.x (2001).

27 Hu, F., Jiang, C., Shen, J., Tang, P. \& Wang, Y. Preoperative predictors for mortality following hip fracture surgery: A systematic review and meta-analysis. Injury 43, 676-685, doi:

10.1016/j.injury.2011.05.017 (2012).

28 Panula, J. et al. Mortality and cause of death in hip fracture patients aged 65 or older - a population-based study. BMC Musculoskelet Disord 12, 1-6, doi: 10.1186/1471-2474-12-105 (2011).

29 Moja, L. et al. Timing matters in hip fracture surgery: patients operated within 48 hours have better outcomes. A meta-analysis and meta-regression of over 190,000 patients. PloS one 7, e46175, doi:10.1371/journal.pone.0046175 (2012).

30 Simunovic, N. et al. Effect of early surgery after hip fracture on mortality and complications: systematic review and meta-analysis. CMAJ 182:1609-16. doi: 10.1503/cmaj.092220 (2010).

31 Grigoryan, K. V., Javedan, H. \& Rudolph, J. L. Orthogeriatric care models and outcomes in hip fracture patients: a systematic review and meta-analysis. J Orthop Trauma. 28, e49 doi: 10.1097/BOT.0b013e3182a5a045 (2014).

32 Prestmo, A. et al. Comprehensive geriatric care for patients with hip fractures: a prospective, randomised, controlled trial. Lancet (London, England) 385, 1623-1633, doi:10.1016/s01406736(14)62409-0 (2015).

33 Leal, J. et al. Impact of hip fracture on hospital care costs: a population-based study. Osteoporos Int. 27:549-58. doi: 10.1007/s00198-015-3277-9 (2016).

34 Neuburger, J. et al. The impact of a national clinician-led audit initiative on care and mortality after hip fracture in England: an external evaluation using time trends in non-audit data. Medical care 53, 686-691, doi:10.1097/mlr.0000000000000383 (2015).

35 Mccain, K. W. Assessing Obliteration by Incorporation: Issues and Caveats. Journal of the American Society for Information Science \& Technology 63, 2129-2139. doi: 10.1002/asi.22719 (2012)

\section{Figures}




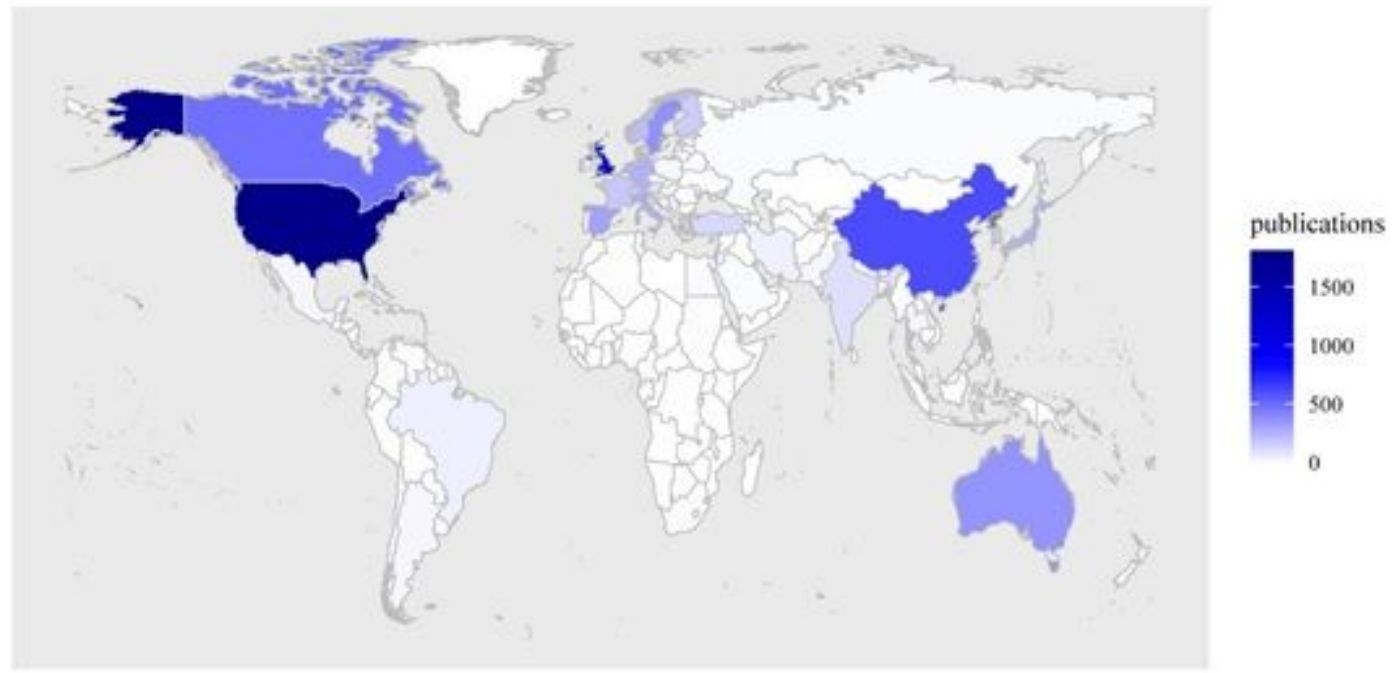

\section{Figure 1}

The distribution world map of hip fracture research. Note: The designations employed and the presentation of the material on this map do not imply the expression of any opinion whatsoever on the part of Research Square concerning the legal status of any country, territory, city or area or of its authorities, or concerning the delimitation of its frontiers or boundaries. This map has been provided by the authors.

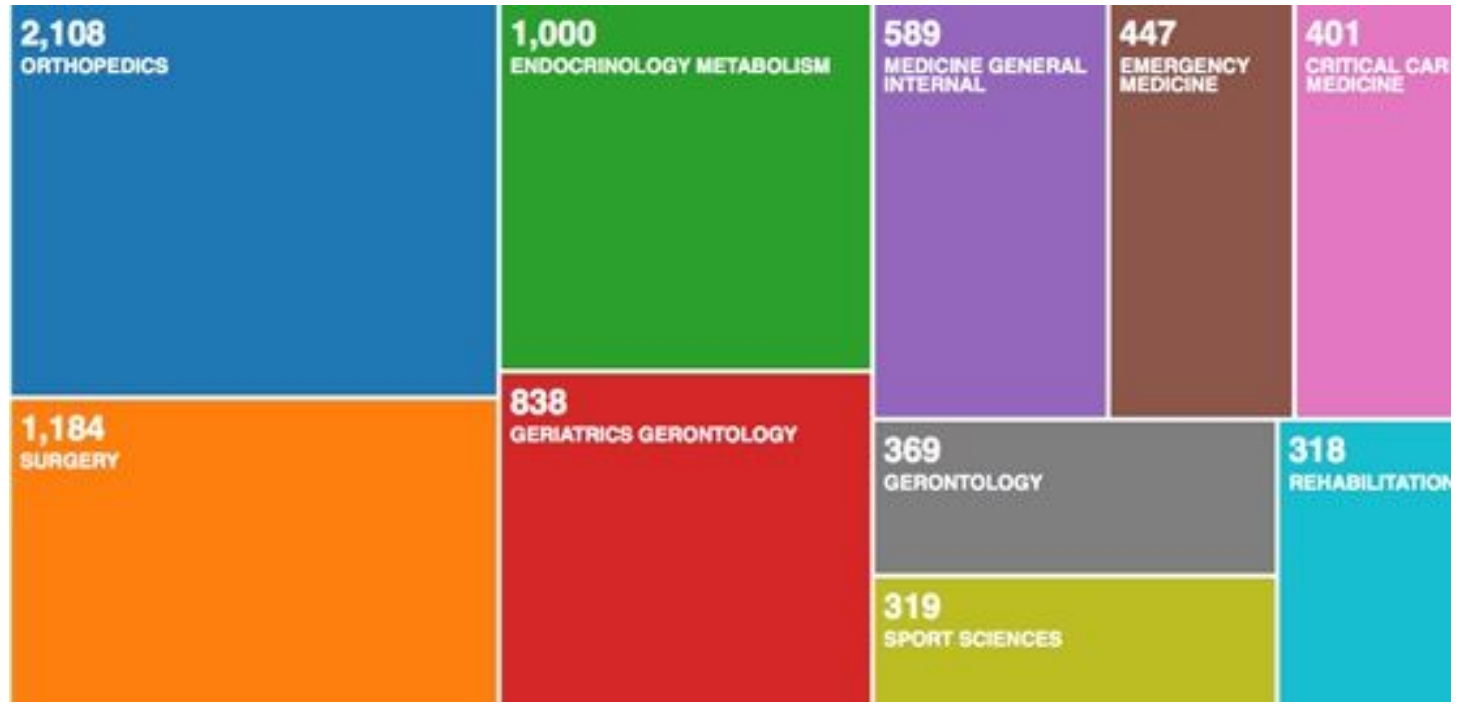

\section{Figure 2}

The top 10 research orientations and the number of publications in each orientation. 


\section{a}
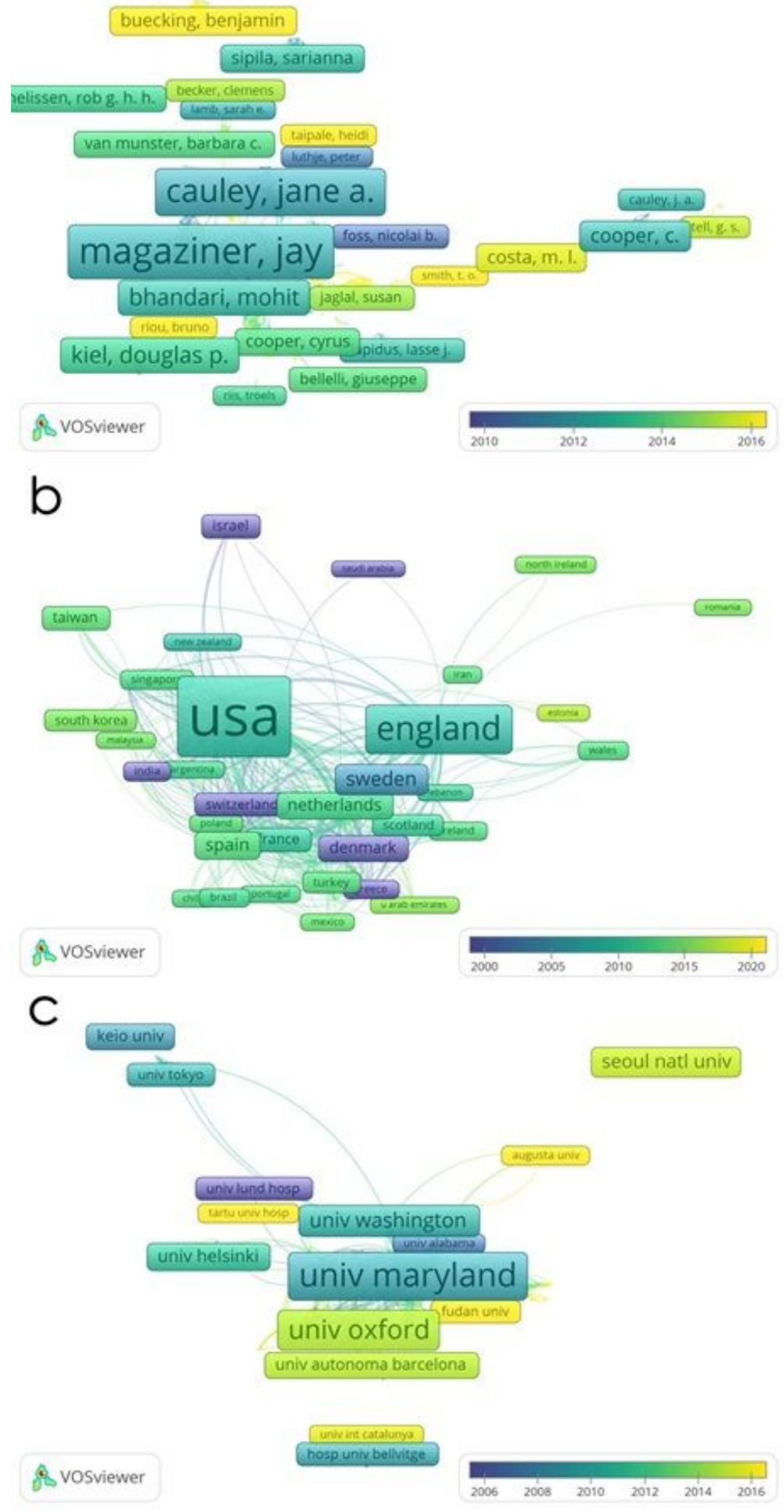

\section{Figure 3}

Coauthorship analysis in hip fracture research. a Mapping of the coauthorship analysis among 480 identified authors. b Mapping of 54 identified countries. c Mapping of 622 institutions. 


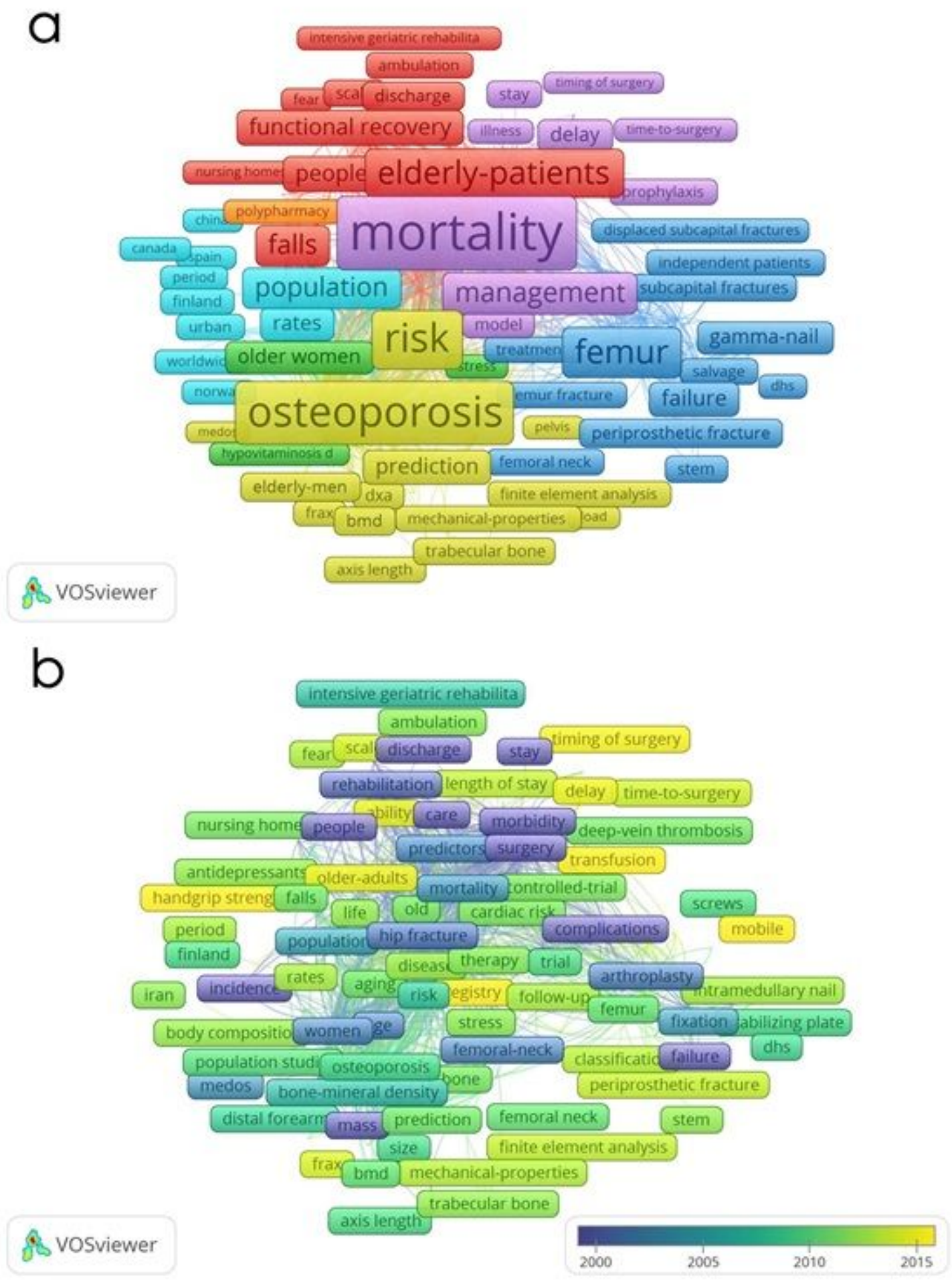

\section{Figure 4}

Co-occurrence analysis on hip fracture research. a Mapping of keywords on hip fracture research; the size of nodes represents the frequency, while the lines between nodes reflect the co-occurrence relationship. A total of 1458 included keywords were divided into 5 clusters: "mortality study" (color purple), "surgical management study" (color deep blue), "rehabilitation study" (color red), and "epidemiology study" (color light blue). $b$ Distribution of keywords according to the time of appearance. The color blue indicates the keywords appeared earlier, whereas the color red reflects the later occurrence. 